\title{
Title: Live attenuated SARS-CoV-2 vaccine candidate: Protective immunity without serious lung lesions in Syrian hamsters
}

Authors: Shinya Okamura ${ }^{1,2}$, Akiho Kashiwabara ${ }^{1,2}$, Hidehiko Suzuki ${ }^{1,2}$, Shiori Ueno ${ }^{3}$, Paola Miyazato ${ }^{1,2}$, Shiro Takekawa ${ }^{2}$, Wataru Kamitani ${ }^{3}$, Koichi Yamanishi ${ }^{2}$ and Hirotaka Ebina ${ }^{1,2,4 *}$

\section{Affiliations:}

${ }^{1}$ Virus vaccine group, BIKEN Innovative Vaccine Research Alliance Laboratories, Institute for Open and Transdisciplinary Research Initiatives, Osaka University, Suita, Osaka, Japan.

${ }^{2}$ The Research Foundation for Microbial Diseases of Osaka University, Suita, Osaka, Japan

${ }^{3}$ Department of Infectious Diseases and Host Defense, Gunma University Graduate School of Medicine, Maebashi, Gunma, Japan

${ }^{4}$ Virus vaccine group, BIKEN Innovative Vaccine Research Alliance Laboratories, Research institute for Microbial Diseases, Osaka University, Suita, Osaka, Japan.

*Correspondence to: hebina@ biken.osaka-u.ac.jp

\begin{abstract}
Various COVID-19 vaccine candidates are currently under clinical trial. However, no live attenuated vaccine has been developed yet, despite their generally high efficacy. Here, we established temperature-sensitive mutant strains of SARS-CoV-2, whose growth was significantly slower than that of the parent strain at $37^{\circ} \mathrm{C}$. One of the strains, A50-18, which presented mutations in nonstructural protein 14 , did not replicate at all at $37^{\circ} \mathrm{C}$ in vitro. In vivo experiments demonstrated that this strain replicated inefficiently in the lungs of Syrian hamsters, and intra-nasal inoculation induced sufficient anti-SARS-CoV-2-neutralizing antibodies to protect against wild type virus infection. These results suggest that the A50-18 strain could be a promising live attenuated vaccine candidate against SARS-CoV-2.
\end{abstract}

One Sentence Summary: A live attenuated virus provided immunity against SARS-CoV-2 in an animal model, making it a promising vaccine candidate.

\section{Main Text:}

A new emerging infectious disease arose in Wuhan, China in 2019; the responsible pathogen was identified as a novel coronavirus (1). This coronavirus strain is related to SARS-CoV, which also emerged in China in 2002, and thus, the novel virus was named SARS-CoV-2, while the infection is known as COVID-19 (2). COVID-19 has been reported to cause pneumonia and lung inflammation, and even more severe symptoms, with elderly people especially vulnerable. To date, approximately 100 million people have been infected globally, and over 2 million people have died of this infectious disease (3).

SARS-CoV-2 is an enveloped positive-sense single-stranded RNA virus that belongs to the Betacoronavirus genus of the Coronaviridae family. The coronavirus genome is approximately $30 \mathrm{~kb}$ long and contains several open reading frames (ORFs) encoding two large polyproteins, 
ORF1a and ORF1ab, and four structural proteins $\square-\square$ spike (S), envelope (E), nucleocapsid (N) and membrane (M) (4). The ORF1ab transcript encodes ORF1a and ORF1b by programmed ribosomal frameshift, and the polyproteins are cleaved to give rise to sixteen nonstructural proteins (NSPs) by the self-cleavage activity of NSP3 and NSP5 proteases $(5,6)$. Among the structural proteins, $\mathrm{S}$ protein, which is a large glycoprotein on the virion surface, attaches to a host cell receptor for entry (7). It was reported that the receptor-binding domain (RBD) in the SARS-CoV-2 S protein binds to human angiotensin converting enzyme 2 (hACE2), thus antiRBD antibodies should neutralize the virus (8). Several vaccine candidates currently in development target the $\mathrm{S}$ protein or its RBD.

Live attenuated vaccines have been developed by various experimental methods. Heterogeneous adaptation is a traditional strategy for isolating attenuated viruses that cannot readily propagate in human cells. For example, the VZV vOka strain was generated by passaging the virus several times in guinea pig embryo fibroblasts (9); the measles virus AIK strain was isolated by passage in sheep kidney cell lines (10-12); and rubella virus vaccine strains were developed by passage in Japanese quail embryo fibroblasts or rabbit kidney cells $(13,14)$. Temperature-sensitive (TS) strains are also selected to create live attenuated vaccines that cannot replicate at body temperature. The AIK-C strain was derived by further passage of the AIK strain in chicken embryo cells at $33^{\circ} \mathrm{C}$. It can replicate at $35^{\circ} \mathrm{C}$, but not at $37^{\circ} \mathrm{C}$ or $39^{\circ} \mathrm{C}(15)$. The rubella virus TO-336 vaccine strain can also replicate at $35^{\circ} \mathrm{C}$, but not at $39^{\circ} \mathrm{C}(16)$. Furthermore, a live attenuated influenza virus vaccine was developed by reassortment of viruses generated from a cold-adapted donor virus, which had temperature-sensitivity-related mutations in six vRNA segments $(17,18)$. This attenuated influenza virus could replicate at $25-33^{\circ} \mathrm{C}$, but not at $37^{\circ} \mathrm{C}$ (18-20). In the case of coronaviruses, it was reported that certain NSP3 mutations in the mouse hepatitis virus (MHV), another member of the Betacoronavirus genus, lead to temperature sensitivity (21).

Here, we generated four TS SARS-CoV-2 strains from a clinical isolate. Among the obtained mutant strains, strain A50-18 showed a unique phenotype with impaired replication at $37^{\circ} \mathrm{C}$ and normal proliferation at $33-35^{\circ} \mathrm{C}$, meaning that it was able to replicate in the nasal cavity but not in the lungs. In vivo analysis in a Syrian hamster model revealed that this strain did not cause lung lesions or weight loss, unlike the parental virus. Furthermore, pre-infection with the novel virus prevented pathogenicity due to parental strain infection. These results suggested that A5018 may be a novel live attenuated vaccine candidate for the prevention of COVID-19.

\section{Results}

\section{Isolation and phenotypic characterization of SARS-CoV-2 temperature-sensitive strains}

To isolate TS strains of SARS-CoV-2, we generated a library of viruses with random mutations from the clinical isolate B-1 virus (Accession number: LC603286). A total of 659 viral plaques were isolated from the library and subjected to screening. For this purpose, Vero cells were infected with all the virus clones and cultured at $32^{\circ} \mathrm{C}$ or $37^{\circ} \mathrm{C}$ and observed daily to evaluate cytopathic effect (CPE). During the process, we selected four TS strains that caused $\mathrm{CPE}$ at $32^{\circ} \mathrm{C}$ 3 days post-infection (dpi), but none at $37^{\circ} \mathrm{C}$.

To analyze the temperature sensitivity in detail, we then evaluated the growth kinetics of the isolated TS strains at $32^{\circ} \mathrm{C}, 34^{\circ} \mathrm{C}$ or $37^{\circ} \mathrm{C}$ (Fig. 1A). Under $32^{\circ} \mathrm{C}$ and $34^{\circ} \mathrm{C}$ conditions, all TS 
strains replicated comparably to the parent B- 1 virus. However, at $37^{\circ} \mathrm{C}$, replication of all TS strains was relatively slower or smaller in scale than the parent strain. The peak viral growth of the H50-11 strain was delayed by 1-2 d compared with the parent B-1 virus, but its maximum viral titer was not significantly different $(p=0.38)$. L50-33 and L50-40 strains hardly proliferated at $37^{\circ} \mathrm{C}$, and the viral titers were less than $10^{4} \mathrm{TCID} 50 / \mathrm{mL}$ even at $5 \mathrm{dpi}$, which is $10^{4}$ times lower than the maximum titer of the B-1 strain. Interestingly, the A50-18 strain showed a unique phenotype, since no infectious viruses were detected in the culture medium at $37^{\circ} \mathrm{C}$ (the TCID50 was under the assay's limit of detection).

Next, these four TS strains were subjected to deep-sequence analysis to identify the mutations involved in the TS phenotype (Table 1). The A50-18 strain had a total of six missense mutations in NSP14, S, E and N proteins. The H50-11 strain showed four missense mutations in NSP3, NSP16 and S proteins. There were two missense mutations in NSP3 in the L50-33 strain and a total of three missense mutations in NSP3 and S proteins in the L50-40 strains were observed. Three of four TS strains had mutations in NSP3. Notably, the mutations located within NSP3 in strains L50-33 and L50-40 were identical, therefore the L445F and K1792R mutation in NSP3 were thought to account for the TS phenotype of these strains. It has been reported that NSP3 mutations in the MAC and PLP2 domains, cause temperature sensitivity in MHV (21). The L445F mutation observed in L50-33 and L50-40 strains is located in the MAC2 domain and, the V404F mutation in H50-11 strain is located very close to this domain of NSP3. These results suggested that these three strains acquired the TS phenotype by a mechanism similar to TS MHV. However, the mutations contributing to the TS phenotype in the A50-18 strain were still unknown.

To identify the mutations involved in temperature sensitivity in the TS strains, we sought to evaluate revertant strains of these viruses. By passaging the TS strains at $37^{\circ} \mathrm{C}$, we obtained several revertants from L50-33 and L50-40 strains, but only one from A50-18 strain (Supplementary Fig. 1). Sequencing results of the latter strain confirmed that the G248V mutation in NSP14 had changed to the wild type sequence, while the other mutations remained (Fig. 2A). In the revertants from L50-33 and L50-40, NSP3 L445F mutation located in the MAC domain mutated either back to leucine as expected, or to cysteine, another amino acid with no aromatic ring (Fig. 2B). To better understand the role of the mutations in NSP14 associated with the TS phenotype of A50-18, we generated a recombinant SARS-CoV-2 with the NSP14 sequence of the TS strain by reverse genetics. Vero cells infected with the recombinant virus were cultured at $32^{\circ} \mathrm{C}$ and $37^{\circ} \mathrm{C}$ and the development of CPE was monitored. Wild type SARSCoV-2 and a recombinant virus with A50-18 envelope, used as control, caused CPE at both $32^{\circ} \mathrm{C}$ and $37^{\circ} \mathrm{C}$. However, the recombinant virus with the A50-18 strain's NSP14 did not cause CPE at $37^{\circ} \mathrm{C}$ (Fig. 2C). These results strongly suggested that the mutations in NSP14 were involved in the temperature sensitivity of A50-18.

\section{Evaluation of pathogenicity}

Temperature sensitive viruses are usually attenuated and have been used as live attenuated vaccines, e.g. measles, rubella and influenza vaccines $(15,18-20)$. Here, we evaluated the pathogenicity of the TS mutant strain A50-18, which showed impaired replication at $37^{\circ} \mathrm{C}$, using Syrian hamsters, widely used as a model for SARS-CoV-2 infection $(22,23)$. Viral pathogenicity was monitored by body weight change after virus infection. An approximate body 
weight decrease of $10 \%$ or $20 \%$ was observed 7 dpi in Syrian hamsters infected with low or high doses of the B-1 virus, respectively (Fig. 3A). In contrast, no body weight loss was observed after infection with the A50-18 strain, even at the high dose.

We investigated acute symptoms resulting from the infection with the TS strain. For that purpose, B-1 or A50-18-infected Syrian hamsters were euthanized 3 dpi, and damage in the lung tissue was evaluated. The lungs of B-1-infected hamsters were larger than those of A50-18infected ones (Fig. 3B, Supplemental Fig. 2). Additionally, apparent bleeding and destruction of alveoli in the lungs of B-1-infected hamsters were detected, but no evident critical tissue damage was observed in those of A50-18-infected hamsters. We also confirmed the amount of virus remaining in the nasal cavity and lungs 3 dpi. The virus titer in nasal wash specimens of B-1and A50-18-infected hamsters was not significantly different, ranges being $4.50 \times 10^{2} \sim 5.40 \times$ $10^{4} \mathrm{PFU} / \mathrm{mL}$ and $6.60 \times 10^{2} \sim 2.60 \times 10^{4} \mathrm{PFU} / \mathrm{mL}$, respectively. On the other hand, A50-18 strain-infected hamsters showed $10^{3}$ times lower virus titer than the B-1-infected group (Fig. $3 \mathrm{C}$ ), the virus titer in lung tissue was $7.00 \times 10^{5} \sim 4.86 \times 10^{6} \mathrm{PFU} / \mathrm{g}$ and $2.00 \times 10^{2} \sim 5.07 \times 10^{3}$ PFU/g in B-1- and A50-18-infected hamsters, respectively. These results suggested that the A5018 strain showed an attenuated phenotype because its replication in lungs was impaired.

\section{Evaluation of antigenicity}

It was reported that SARS-CoV-2 infection induces immunity and prevents reinfection (22). Thus, we sought to evaluate whether the attenuated A50-18 strain could be used as a live vaccine to protect against wild type viral infection. Syrian hamsters were inoculated intranasally with the B-1 virus or the attenuated A50-18 strain and reinfected with the wild type B-1 virus $21 \mathrm{~d}$ after the first infection (Fig. 4A). Vaccine efficacy was assessed by body weight change. Primary B-1 strain infection resulted in a noticeable body weight loss in naïve hamsters, but no significant weight decrease was observed in the hamsters pre-infected with B-1 or A50-18 (Fig. 4B), suggesting the induction of protective immunity. The titer of neutralizing antibodies in the serum of mock-infected hamsters was <20 (Fig. 4C). Infection with B-1 or A50-18 strains induced neutralizing antibodies, titers being $640-5120$ or $160-640$, respectively. Neutralizing antibodies induced by the attenuated A50-18 strain infection were sufficient to prevent viral reinfection in Syrian hamsters.

\section{Discussion}

In this study, we isolated new TS SARS-CoV-2 strains. These strains showed various levels of temperature sensitivity at $37^{\circ} \mathrm{C}$. The $\mathrm{H} 50-11$ strain replicated slower than the wild type B-1 virus, while L50-33 and L50-40 strains barely replicated at $37^{\circ} \mathrm{C}$. These strains presented missense mutations in NSP3 MAC2 and Y domains. It was reported that mutations in NSP3 MAC or PLP domains can cause temperature sensitivity in MHV, likely by enhancing proteasome-mediated degradation (21). Therefore, these strains probably obtained the TS phenotype by a mechanism similar to the previously reported MHV TS mutants.

In contrast, A50-18 strain replication was not observed at $37^{\circ} \mathrm{C}$ for $5 \mathrm{~d}$, although it replicated similarly to the B-1 wild type virus at $32^{\circ} \mathrm{C}$ and $34^{\circ} \mathrm{C}$. We revealed that the NSP14 protein of A50-18, which presented three mutations that have not yet been reported, contributed to the temperature sensitivity of the strain. It is known that NSP14 has exo-ribonuclease and N7 methyltransferase domains and is involved in viral genome replication by forming a complex 
with other NSPs (24). The G248V mutation observed in the A50-18 strain is located near the catalytic residue D243, that confers the exo-ribonuclease activity (25). The G416S mutation is located near motif IV of the methyltransferase domain (26). These mutations probably involve temperature-dependent enzymatic activity or temperature-dependent structural differences in NSP14 that affect its ability to form a complex with other NSPs. Since the G248V mutation was the only one that back mutated in the revertant strain, it is likely that it is critically involved in this phenotype, although the significance of the other mutations, alone or in combination, still needs to be clarified.

S. H. Seo et al. previously reported a cold-adapted SARS-CoV-2 strain as a vaccine candidate. (27) The replication of the cold-adapted strain was reduced at $37^{\circ} \mathrm{C}$ but still clearly evident, as it was observed for the H50-11 strain described in the current study. In contrast, the A50-18 strain showed a sharp TS phenotype of impaired replication at $37^{\circ} \mathrm{C}$. In vivo experiments demonstrated that this strain did not replicate efficiently in the lungs, evidenced by the lack of lung tissue damage or weight decrease in hamsters infected with this strain, making it a safer candidate for a live attenuated vaccine. The measles virus AIK-C strain and influenza virus Ann Arbor/1/60 and Ann Arbor/1/66 strains, which are live attenuated vaccine strains approved for clinical use, showed replication under $33^{\circ} \mathrm{C}$ or $35^{\circ} \mathrm{C}$ culture conditions, but not at $37^{\circ} \mathrm{C}(15,18-20)$. The A5018 strain presents a TS phenotype similar to live attenuated vaccines already in use in clinical settings.

Live attenuated vaccines can generate concerns regarding their safety, since the risks of reverting to a pathogenic form and adverse reactions remains (28). However, since they can induce an effective and long-lasting immune response that is nearly as good as the one induced by the wild type virus, they have been used since the 1780's, with Jenner's smallpox vaccine (29). For the influenza virus, it was previously reported that an attenuated TS reassortant virus induced $\operatorname{IgA}$ secretion in the nasal cavity (30) with neutralizing activity, providing protection against infection (31). Additionally, a live attenuated influenza virus vaccine could induce specific CD8+ T cell immunity (32). Like the influenza virus, SARS-CoV-2 is thought to be transmitted through the nasal cavity where epithelial cells express high levels of the receptor ACE2 (33). In addition, it was reported that dimeric $\operatorname{IgA}$ in mucosal secretions showed an enhanced neutralizing activity against SARS-CoV-2 (34). A50-18 replicated in the nasal cavity and the upper respiratory tract, which exhibit a temperature lower than the lower respiratory tract and lungs. Therefore, intranasal inoculation with a TS SARS-CoV-2 strain may also induce the production of IgA and specific $\mathrm{T}$ cells in this compartment. However, neurological manifestations in COVID-19 have been the focus of great concern (35), and the intra-nasal administration of live attenuated SARSCoV-2 strains could trigger the development of taste and olfactory disorders, already reported to be related to this virus (36). The accurate evaluation of these manifestations should be more thoroughly tested in non-human primate models.

In this study we report the isolation of a new SARS-CoV-2 TS strain (A50-18) that showed lower pathogenicity than the wild type strain and induced sufficient protective immunity. Overall, this novel TS strain should be promising as a live attenuated vaccine against SARSCoV-2.

References and Notes: 
1. N. Zhu et a1., A Novel Coronavirus from Patients with Pneumonia in China, 2019. N Eng1 $J$ Med 382, 727-733 (2020).

2. P. Zhou et al., A pneumonia outbreak associated with a new coronavirus of probable bat origin. Nature 579, 270-273 (2020).

5 3. Weekly epidemiological update - 27 January 2021. WHO, (2021).

4. S. Perlman, J. Netland, Coronaviruses post-SARS: update on replication and pathogenesis. Nat Rev Microbiol 7, 439-450 (2009).

5. J. Lei, Y. Kusov, R. Hilgenfeld, Nsp3 of coronaviruses: Structures and functions of a large multi-domain protein. Antiviral Res 149, 58-74 (2018).

106 6. Thiel et al., Mechanisms and enzymes involved in SARS coronavirus genome expression. J Gen Virol 84, 2305-2315 (2003).

7. F. Li, Structure, Function, and Evolution of Coronavirus Spike Proteins. Annu Rev Virol 3, 237-261 (2016).

8. J. Shang et al., Cell entry mechanisms of SARS-CoV-2. Proc Nat1 Acad Sci U S A 117, 11727-11734 (2020).

9. M. Takahashi, T. Otsuka, Y. Okuno, Y. Asano, T. Yazaki, Live vaccine used to prevent the spread of varicella in children in hospital. Lancet 2, 1288-1290 (1974).

10. C. L. Parks et al., Comparison of predicted amino acid sequences of measles virus strains in the Edmonston vaccine lineage. J Virol 75, 910-920 (2001).

11. S. Makino, K. Sasaki, F. Nazari, M. Nakagawa, N. Nakamura, Cultivation of measles virus in sheep kidney cells. Jpn J Microbiol 14, 501-504 (1970).

12. K. Sasaki, Studies on the modification of the live AIK measles vaccine. I. Adaptation of the further attenuated AIK measles virus (the strain AIK-L33) to chick embryo cells. Kitasato Arch Exp Med 47, 1-12 (1974).

13. A. Shishido, M. Ohtawara, Development of attenuated rubella virus vaccines in Japan. Jpn J Med Sci Biol 29, 227-253 (1976).

14. L. A. Zimmerman, S. E. Reef, W. A. Orenstein, Rubella Vaccine-A Tale of Appropriate Caution and Remarkable Success. JAMA Pediatr 172, 95-96 (2018).

15. K. Komase et al., The phosphoprotein of attenuated measles AIK-C vaccine strain

16. K. Okamoto et al., Analysis of the temperature sensitivity of Japanese rubella vaccine strain T0-336. vac and its effect on immunogenicity in the guinea pig. Virology 491, 89-95 (2016).

17. H. F. Maassab, M. L. Bryant, The development of live attenuated cold-adapted influenza virus vaccine for humans. Rev Med Virol 9, 237-244 (1999).

18. B. R. Murphy, K. Coelingh, Principles underlying the development and use of live attenuated cold-adapted influenza A and B virus vaccines. Viral Immunol 15, 295-323 (2002).

19. N. J. Cox, F. Kitame, A. P. Kenda1, H. F. Maassab, C. Naeve, Identification of sequence changes in the cold-adapted, live attenuated influenza vaccine strain, A/Ann Arbor/6/60 (H2N2). Virology 167, 554-567 (1988).

20. A. Cox, S. Dewhurst, A Single Mutation at PB1 Residue 319 Dramatically Increases the Safety of PR8 Live Attenuated Influenza Vaccine in a Murine Model without Compromising Vaccine Efficacy. J Virol 90, 2702-2705 (2015).

45 21. X. Deng et al., Analysis of Coronavirus Temperature-Sensitive Mutants Reveals an Interplay between the Macrodomain and Papain-Like Protease Impacting Replication and Pathogenesis. J Virol 93, (2019).

22. M. Imai et a1., Syrian hamsters as a small animal model for SARS-CoV-2 infection and countermeasure development. Proc Nat1 Acad Sci U S A 117, 16587-16595 (2020). 
23. S. F. Sia et al., Pathogenesis and transmission of SARS-CoV-2 in golden hamsters. Nature 583, 834-838 (2020).

24. N. S. Ogando et al., The Enzymatic Activity of the nsp14 Exoribonuclease Is Critical for Replication of MERS-CoV and SARS-CoV-2. J Virol 94, (2020).

25. Y. Ma et a1., Structural basis and functional analysis of the SARS coronavirus nsp14nsp10 complex. Proc Nat1 Acad Sci US A 112, 9436-9441 (2015).

26. N. S. Ogando et a1., The Curious Case of the Nidovirus Exoribonuclease: Its Role in RNA Synthesis and Replication Fidelity. Front Microbiol 10, 1813 (2019).

27. S. H. Seo, Y. Jang, Cold-Adapted Live Attenuated SARS-Cov-2 Vaccine Completely Protects Human ACE2 Transgenic Mice from SARS-Cov-2 Infection. Vaccines (Base1) 8, (2020).

28. 0 . Kew et al., Outbreak of poliomyelitis in Hispaniola associated with circulating type 1 vaccine-derived poliovirus. Science 296, 356-359 (2002).

29. S. Riedel, Edward Jenner and the history of smallpox and vaccination. Proc (Bayl Univ Med Cent) 18, 21-25 (2005).

30. M. L. Clements, B. R. Murphy, Development and persistence of local and systemic antibody responses in adults given live attenuated or inactivated influenza A virus vaccine. J Clin Microbiol 23, 66-72 (1986).

31. Y. Asahi-Ozaki et al., Secretory IgA antibodies provide cross-protection against infection with different strains of influenza B virus. J Med Virol 74, 328-335 (2004).

32. R. Bodewes et al., Annual vaccination against influenza virus hampers development of virus-specific CD8 ${ }^{+}$T cell immunity in children. J Virol 85, 11995-12000 (2011).

33. W. Sungnak et al., SARS-CoV-2 entry factors are highly expressed in nasal epithelial cells together with innate immune genes. Nat Med 26, 681-687 (2020).

34. Z. Wang et a1., Enhanced SARS-CoV-2 neutralization by dimeric IgA. Sci Trans1 Med 13, (2021).

35. B. Fiani, C. Covarrubias, A. Desai, M. Sekhon, R. Jarrah, A Contemporary Review of Neurological Sequelae of COVID-19. Front Neurol 11, 640 (2020).

36. S. Urata et al., Regeneration Profiles of 01factory Epithelium after SARS-CoV-2 Infection in Golden Syrian Hamsters. ACS Chem Neurosci, (2021).

Acknowledgments: We appreciate the assistance from Mitsuyo Kosaka (BIKEN). The authors acknowledge the NGS core facility of the Genome Information Research Center at the Research Institute for Microbial Diseases of Osaka University for their support with RNA-seq. WK521 strain of SARS-CoV-2 was kindly provided by Shutoku Matsuyama (diagnosis group in NCGM and NIID).

Funding: This study was supported by BIKEN.

Author contributions: H.E. conceived and designed the study. S.O. conducted the majority of the experiments. A.K. performed the isolation of TS mutants and measured the virus titers. H.S. performed in vivo experiments. W.K. and S.U. constructed the recombinant infectious clone of SARS-CoV-2. S.O. and P.M. wrote the original manuscript and H.E. revised the original manuscript. S.T., K.Y., and H.E. supervised the project. 
Competing interest: S.O., A.K., H.S., P.M., S.T., K.Y., and H.E. are employed by BIKEN. We report that S.O., A.K., and H.E. are named on a patent that describes the use of the TS mutants as vaccines, currently being filed by BIKEN. W.K. and S.U. have no conflict of interests to declare.

Data and materials availability: RNA-sequencing data of the SARS-CoV-2 clinical isolate and the TS mutants were deposited at DDBJ (Accession numbers for B-1, A50-18, H50-11, L50-33 and L50-40 are LC603286, LV603287, LC603288, LC603289 and LC603290, respectively). All other data needed to evaluate the conclusions in the paper are present in the paper or the Supplementary Materials.

\section{Supplementary Materials:}

Materials and Methods

Figures S1-S2

\section{Fig. 1. Growth kinetics and missense mutations of TS strains}

The growth kinetics of four TS (temperature-sensitive) mutants at different temperatures is shown, compared to the WT (B-1) virus. Vero cells were infected with $1 \times 104$ TCID50 SARS$\mathrm{CoV}-2$ strains (approximate $\mathrm{MOI}=0.01$ ) and incubated at the indicated temperatures. The virus titer of the supernatant was evaluated by TCID50. Symbols represent the average of three independent experiments, and error bars mean SD.

Fig. 2. Identification of the mutations involved in temperature sensitivity in the TS strains

(A, B) Details of the amino acid sequence in segments of NSP14 (A) and NSP3 (B) of revertant viruses (from A50-18, L50-33 and L50-40) are shown. Differences with the B-1 virus are highlighted. (C) Development of CPE by infection with recombinant SARS-CoV-2 viruses, containing the NSP14 or envelope genes of the A50-18 strain (r-NSP14 ${ }_{\mathrm{A} 50-18}, \mathrm{r}_{-\mathrm{Env}} \mathrm{A50-18}$, respectively). Reconstituted viruses were added to Vero cells and incubated at $32^{\circ} \mathrm{C}$ (left column). Subsequently, these supernatants were transferred to new Vero cells, and cultured at $32^{\circ} \mathrm{C}$ or $37^{\circ} \mathrm{C}$ (middle and right columns, respectively). CPE was confirmed by crystal violet staining.

\section{Fig. 3. Temperature-sensitive A50-18 is an attenuated SARS-CoV-2 strain}

(A) Weight change of the Syrian hamsters infected with the A50-18 strain or B-1 virus. The average weight change is plotted, and error bars represent the SD. (B) Representative images of H\&E (hematoxylin \& eosin) staining of lung tissue sections are shown. (C) The viral titer of nasal wash specimens and lung homogenates at three days post-infection were evaluated by plaque formation assay. Bars depict the median values, and symbols represent individual viral titers. Error bars represent mean SDs. For statistical analysis, one-way ANOVA was performed. n.s., not significant.

\section{Fig. 4. A50-18 strain induces protective immunity in Syrian hamsters}

(A) Overview of the re-challenge protocol is illustrated. (B) Weight change in Syrian hamsters after B-1 virus re-challenge. The average weight change is plotted and error bars represent mean 
SDs. (C) Neutralizing antibody titers of the sera from B-1- or A50-18-infected hamsters were evaluated with the authentic SARS-CoV-2 B-1 virus. Median values are plotted, and error bars represent the SDs. i.n., intra-nasal. 
bioRxiv preprint doi: https://doi.org/10.1101/2021.02.15.430863; this version posted February 16, 2021. The copyright holder for this preprint (which was not certified by peer review) is the author/funder. All rights reserved. No reuse allowed without permission.

\section{Fig. 1}
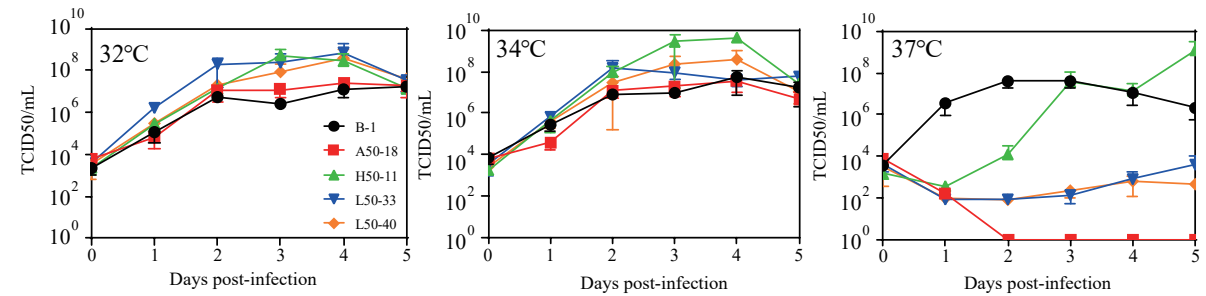
bioRxiv preprint doi: https://doi.org/10.1101/2021.02.15.430863; this version posted February 16, 2021. The copyright holder for this preprint (which was not certified by peer review) is the author/funder. All rights reserved. No reuse allowed without permission.

Fig. 2

A

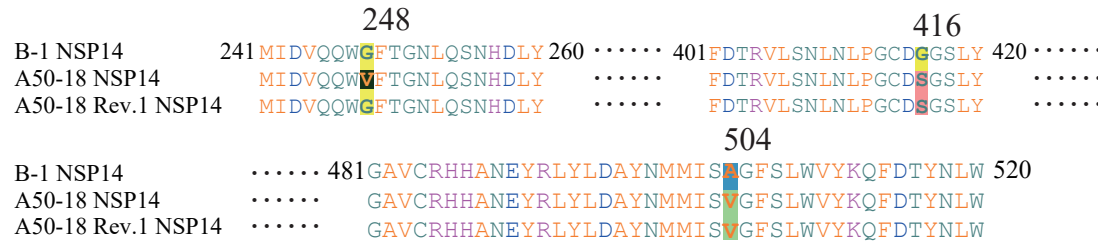

B

B-1 NSP3

L50-33 NSP3

L50-40 NSP3

L50-33 Rev.1 NSP3

L50-33 Rev. 2 NSP3

L50-40 Rev. 1 NSP3

L50-40 Rev.2 NSP3
445

441 INGNLHPDSATLVSDIDITE 460 INGNFHPDSATLVSDIDITH INGNGHPDSATLVSDIDITE INGNCHPDSATLVSDIDITF INGNLHPDSATLVSDIDITH ITGNLHPDSATLVSDIDITH INGN匹HPDSATLVSDIDITE
1792

1781 SSTENVPMEKLKTLVATAEA 1800

..... SSTFNVPMEKIRTLVATAEA

..... SSTFNVPMEKTRTIVATAEA

..... SSTENVPMEKLRTLVATAEA

S.... STENVMMEKLRTLVATAEA

..... SSTFNVPMEKLRTIVATAEA

..... SSTFNVPMEKIRTLVATAEA

$\mathrm{C}$

$32^{\circ} \mathrm{C}$

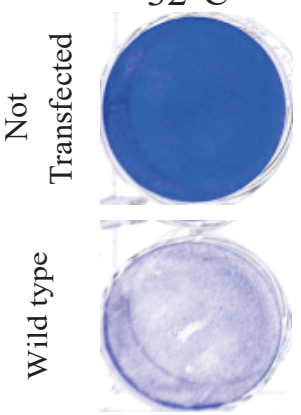

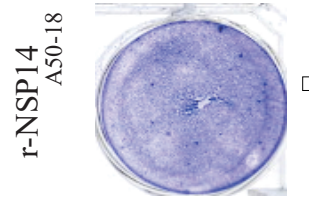

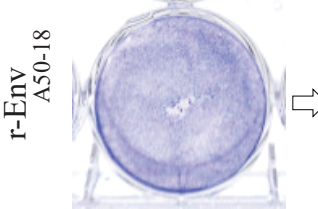

$32^{\circ} \mathrm{C}$

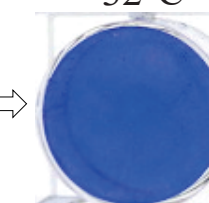

$37^{\circ} \mathrm{C}$
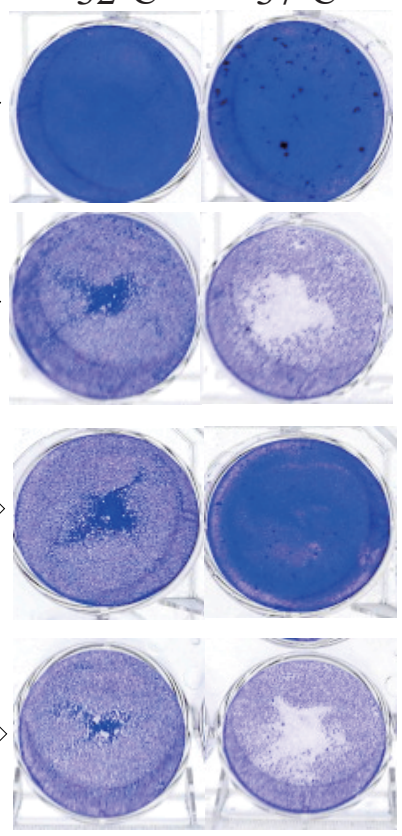
Fig. 3

A

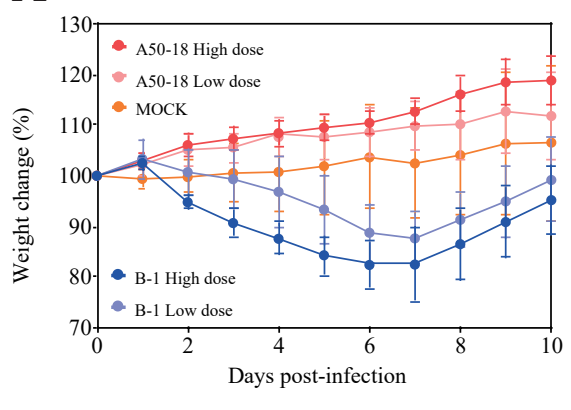

B

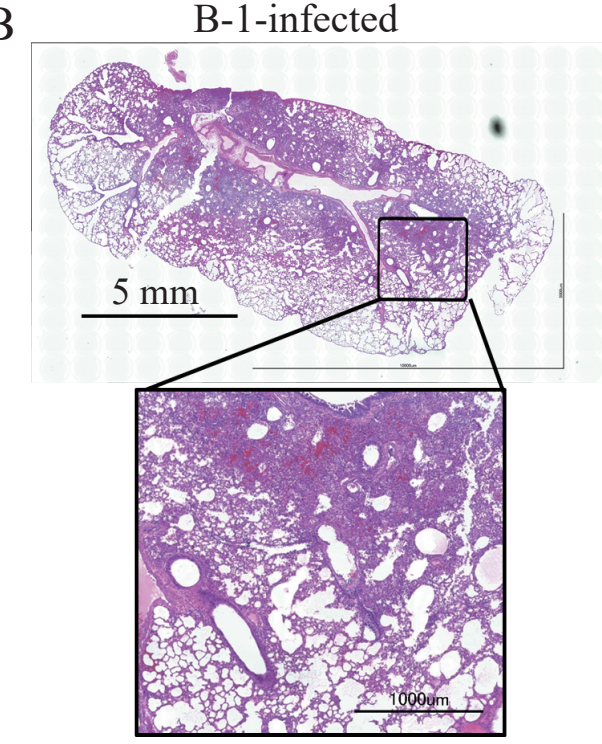

$\mathrm{C}$

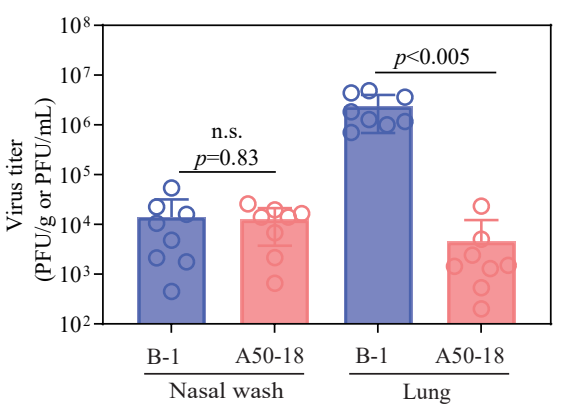

A50-18-infected

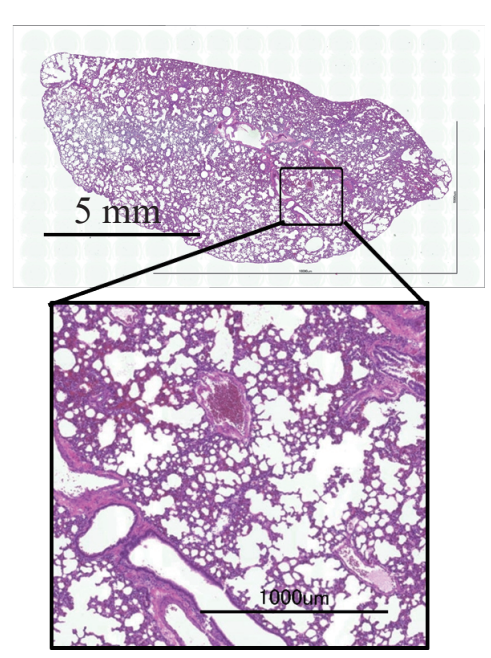


Fig. 4

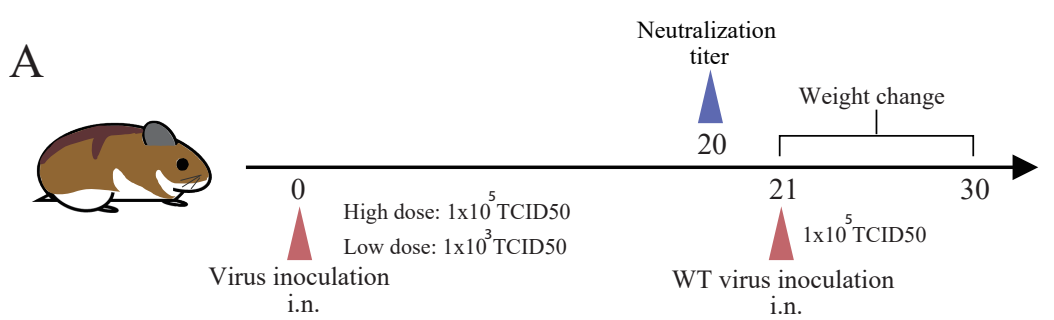

$\mathrm{B}$

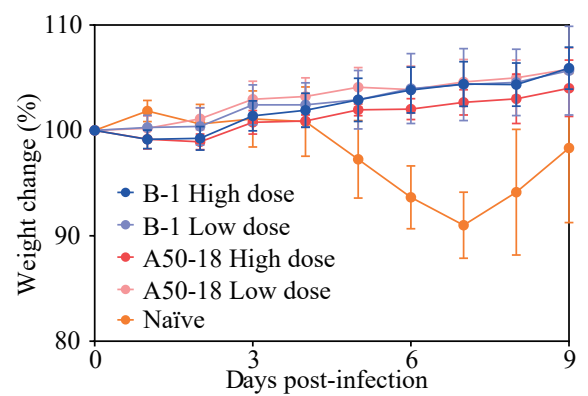

$\mathrm{C}$

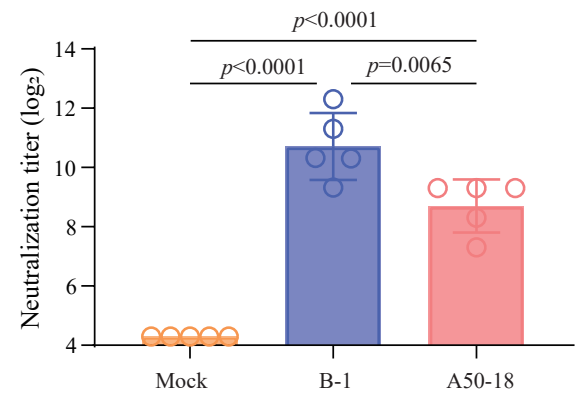


Table 1. Missense mutations in temperature-sensitive mutants

\begin{tabular}{cccc}
\hline Strain & $\begin{array}{c}\text { Nucleotide } \\
\text { substitution }\end{array}$ & Protein & $\begin{array}{c}\text { Amino acid } \\
\text { substitution }\end{array}$ \\
\hline \multirow{2}{*}{ A50-18 } & G18782T & & G248V \\
& G19285A & NSP14 & G416S \\
& C19550T & & A504V \\
& C24198T & Spike & A879V \\
& T26327C & Envelope & L28P \\
& C28278T & Nucleocapsid & S2F \\
\hline \multirow{2}{*}{ H50-11 } & T3930C & NSP3 & V404A \\
& G8213A & D1832N \\
& G20857A & NSP16 & V67I \\
\hline \multirow{2}{*}{ L50-33 } & C23778A & Spike & T739K \\
\hline & C4052T & NSP3 & L445F \\
& A8094G & & K1792R \\
\hline & C4052T & NSP3 & L445F \\
& A8094G & Spike & L54W \\
\hline
\end{tabular}

Missense mutations compared to the Wuhan strain (NC045512) are indicated. 